\title{
Parasitoses intestinais e a inter-relação com os aspectos socioeconômicos de indivíduos residentes em um povoado rural (Rosápolis de Parnaíba-PI)
}

Intestinal parasites and their interrelationship with socioeconomic aspects of individuals living in a rural community

\author{
M. L. Viana ${ }^{1}$; N. R. Fialho'; S. M. S. Rocha ${ }^{1}$; T. C. L. A. Alves ${ }^{1}$; R. A. Trindade 2 \\ e A. C. F. L. Melo ${ }^{3 *}$ \\ ${ }^{1}$ Departamento de Biomedicina - Campus de Parnaíba da Universidade Federal do Piauí (UFPI), 64202-020, \\ Parnaíba, PI, Brasil \\ ${ }^{2}$ Departamento de Análises Clínicas e Toxicológicas - Faculdade de Farmácia da Universidade Federal do Rio de \\ Janeiro (UFRJ),21941-902, Rio de Janeiro, RJ, Brasil \\ ${ }^{3}$ Departamento de Patologia e Medicinal Legal - Faculdade de Medicina da Universidade Federal do Ceará (UFC), \\ 60430-160, Fortaleza, CE, Brasil
}

*acflmelo@gmail.com

(Recebido em 03 de abril de 2017; aceito em 15 de agosto de 2017)

\begin{abstract}
As parasitoses intestinais ainda são problemas de saúde pública devido às altas taxas de morbidade e associação às precárias condições de saneamento e hábitos higiênicos da população. Em comunidades rurais, essas infecções são agravadas pela falta de diagnóstico e tratamento adequado. Portanto, os objetivos deste trabalho foram determinar a prevalência das parasitoses intestinais na comunidade de Rosápolis de ParnaíbaPI e analisar os aspectos socioeconômicos associados. Amostras fecais $(n=1377)$ coletadas de indivíduos em 360 residências foram analisadas pelo método de Hoffman. Paralelamente, informações socioeconômicas e de hábitos higiênicos foram obtidas por um questionário aplicado ao responsável pelo domicílio. Os resultados das análises laboratoriais revelaram uma prevalência global de enteroparasitoses de 53,9\% (743/1377). Entre os parasitos encontrados, protozoários e helmintos corresponderam a 70,3\% e 15,3\%, respectivamente. Infecções mistas (protozoários+helmintos) foram encontradas em 14,2\% dos parasitados. Giardia duodenalis foi o protozoário patogênico mais prevalente $(25,6 \%)$. Entre helmintos, Ascaris lumbricoides $(18,8 \%)$ e ancilostomídeo $(12,6 \%)$ foram os mais encontrados. Não houve diferença estatisticamente significativa quanto ao gênero ou idade dos indivíduos em relação à prevalência. Aspectos socioeconômicos e higiênico-sanitários, tais como consumo de água de torneira sem tratamento (filtração ou fervura) e consumo de vegetais crus e/ou lavados com água da torneira, além de uma alta taxa de "queima" como destino final do lixo, foram os hábitos mais relatados pelos indivíduos. Conclui-se pela alta prevalência de enteroparasitoses nos indivíduos residentes do povoado Rosápolis que essas infecções ainda constituem um importante grupo de doenças preveníveis que afetam as comunidades rurais.
\end{abstract}

Palavras-chave: Doenças parasitárias, Epidemiologia, População rural.

Intestinal parasites remain as relevant public health problems. Parasitic infections can cause high morbidity rates and, in general, they are associated with precarious sanitation and hygienic habits of population. In rural communities, these infections can be aggravated by lack of proper diagnosis and treatment. Therefore, this study aimed to determine the prevalence of intestinal parasites in individuals living at Rosápolis community, located in the city of Parnaíba-PI, followed by analysis of their associated socioeconomic conditions. A total of 1377 fecal samples collected from 360 residences were analyzed by the spontaneous-sedimentation method of Hoffman. At the same time, socioeconomic and hygienic habit data were obtained using objective questionnaires applied in each residence. The overall prevalence of enteroparasitosis was 53.9\% (743/1377). Among positive samples, protozoa and helminths corresponded to $70.3 \%$ and $15.3 \%$, respectively. Mixed infections consisting of protozoa and helminths were found in 14.2\%. Giardia duodenalis was the most prevalent pathogenic protozoan (25.6\%), whereas Ascaris lumbricoides (18.8\%) and ancylostomide (12.6\%) were the most common helminths. Gender or age showed no statistically significant differences in relation to prevalence. Hygienic-sanitary habits and socioeconomic aspects, i.e., consumption of untreated tap water (either by filtration or boiling), consumption of raw and/or tap water washed vegetables, and high rates of "burning" as final destination of domestic wastes were the most reported habits by residents. In conclusion, 
the high prevalence of enteroparasitosis found in individuals living at Rosápolis community has indicated that these infections remain as important preventable diseases affecting this rural community.

Keywords: Epidemiology, Parasitic diseases, Rural communities.

\section{INTRODUÇÃO}

Doenças parasitárias, particularmente aquelas causadas por enteroparasitos, ainda constituem um grupo importante de infecções humanas preveníveis que, embora apresentem baixo potencial de mortalidade, provocam eventos transitórios importantes de morbidade, tais como deficiência na absorção intestinal de nutrientes, diarreias e anemias, podendo ainda ocasionar limitações crônicas ao desenvolvimento intelectual, produtivo e social [1-4]. Comumente, as infecções por enteroparasitos estão associadas às precárias condições higiênico-sanitárias sob as quais estão submetidos os indivíduos parasitados. Entretanto, em países ainda em desenvolvimento como o Brasil, que apresenta discrepantes condições socioeconômicas, os estudos de prevalência têm revelado altas taxas de indivíduos infectados por parasitas intestinais que não estão restritas às regiões mais pobres [2, 5-9]. Fatores sociais, tais como a falta de saneamento básico adequado, fornecimento de água tratada, dificuldade de acesso ao diagnóstico médico e ao tratamento eficiente, propiciam a manutenção das altas prevalências, principalmente em regiões menos assistidas por políticas públicas, por exemplo, assentamentos, aldeias indígenas, comunidades ribeirinhas, zonas rurais e quilombolas [2, 8, 10-13]. No Brasil, as doenças de transmissão oralfecal, especialmente aquelas causadoras de eventos diarreicos como as enteroparasitoses e as infecções por enterobactérias patogênicas, representam, em média, mais de $80 \%$ das patologias relacionadas ao saneamento ambiental inadequado [14].

Além dos fatores socioeconômicos e culturais que afetam a nutrição dos indivíduos, a permanência das infecções por enteroparasitos, seja por falta de diagnóstico, reinfecção ou tratamento inadequado, também interfere na absorção dos nutrientes, induz sangramentos intestinais e reduz a ingestão alimentar [15]. Em conjunto, esses fatores podem causar desnutrições graves e complicações significativas, tais como obstrução intestinal, prolapso retal, anemias severas e formação de abscessos extraintestinais, principalmente hepáticos, em casos de invasão das mucosas intestinais [16].

No estado do Piauí, os levantamentos epidemiológicos já realizados em diferentes grupos populacionais têm revelado sempre altas prevalências de enteroparasitoses [13, 17-19]. Além disso, esses estudos têm mostrado que fatores como depósito irregular de lixo próximo ao domić́lio, ausência parcial ou total de saneamento básico e falta de conhecimento dos hábitos higiênicosanitários adequados são frequentemente relatados pelos indivíduos participantes dos estudos [13, 17-19]. Em relação à falta de conhecimento da população sobre hábitos higiênicos adequados, é importante ressaltar que a educação sanitária da população também contribui para reduzir as infecções parasitárias [20]. Ainda que nem sempre seja possível comprovar estatisticamente as associações entre esses fatores e as altas taxas de prevalências, relatos semelhantes dessas condições são corroborados por outros estudos epidemiológicos similares em outras regiões do país [21-23].

Neste contexto, o objetivo do presente trabalho foi determinar a prevalência global de enteroparasitos e descrever as espécies mais encontradas nos indivíduos infectados residentes do povoado rural de Rosápolis, situado no município de Parnaíba, litoral do estado do Piauí. Paralelamente, também são descritos os principais aspectos socioeconômicos e higiênico-sanitários relatados pelos residentes nos domicílios integrantes do referido povoado, a fim de verificar quais desses aspectos estão associados ao maior risco de transmissão e permanência das infecções parasitárias.

\section{MATERIAL E MÉTODOS}

As amostras fecais foram coletadas no povoado de Rosápolis, localizado no município de Parnaíba, estado do Piaú, no período de setembro de 2012 a setembro de 2013. No total, 1377 amostras de fezes, sendo 628 amostras de indivíduos do sexo masculino e 749 amostras de 
indivíduos do sexo feminino, foram coletadas e devidamente acondicionadas em coletores plásticos descartáveis contendo como fixador solução de formol a 10\%. Os coletores foram devidamente identificados e transportados ao Laboratório de Parasitologia Clínica do Campus de Parnaíba da Universidade Federal do Piaú.

As amostras fecais foram submetidas à análise microscópica para pesquisa laboratorial de parasitos através do método de Hoffman (1934) [24] que tem como princípio a sedimentação espontânea das fezes. Neste método, uma pequena porção das fezes é homogeneizada com água e transferida por filtração em gaze dobrada para um cálice de sedimentação. Após a filtração, o cálice é completado com água até próximo à borda superior e o conteúdo novamente homogeneizado com bastão de vidro. O material é deixado em repouso no cálice de sedimentação por no mínimo 2 horas. Após este tempo, uma pequena alíquota do sedimento da amostra no fundo do cálice é retirada, depositada sobre lâmina de microscopia e coberta com lamínula. Em seguida, a lâmina contendo o material fecal é submetida à análise microscópica em aumento de 100 e 400 vezes.

Os laudos contendo os resultados das análises parasitológicas de fezes foram encaminhados ao posto de saúde do povoado para que os indivíduos ou responsáveis legais os retirassem e, juntamente, recebessem as orientações médicas para o tratamento nos casos de resultados positivos para parasitos.

No povoado de Rosapólis foram visitadas 360 residências, sendo aplicado um questionário socioeconômico e higiênico-sanitário por residência. As residências foram selecionadas por meio de uma intervenção em saúde realizada pelo posto de saúde da região em conjunto com a Universidade Federal do Piauí, Campus Ministro Reis Velloso em Parnaíba. O questionário aplicado constituiu-se de questões objetivas de múltipla escolha referentes às condições socioeconômicas e higiênico-sanitárias das residências e seus habitantes.

A realização deste estudo foi aprovada pelo Comitê de Ética em Pesquisa da Universidade Federal do Piauí, registrado sob n ${ }^{\circ}$ CAAE 0014.0.045.000-09. Cada participante foi devidamente informado sobre os propósitos específicos da pesquisa e foi firmado entre as partes um termo de consentimento autorizando a utilização do material coletado.

Os resultados obtidos foram organizados em tabelas de contingência $2 \times 2$ relacionando a prevalência global de indivíduos parasitados, sexo e faixa etária. Os valores foram avaliados pelo teste do qui-quadrado quanto à significância estatística assumindo uma probabilidade de erro de $5 \%$ ( $p$-valor <0,05). Para as prevalências específicas, tais como, classificação e espécies de parasitos, foram determinados os intervalos de confiança (IC) no nível de 95\% de probabilidade de ocorrência na população estudada.

\section{RESULTADOS}

Das 1377 amostras coletadas e analisadas por microscopia de luz, 743 (53,9\%) encontravam-se positivas. A Tabela 1 apresenta a distribuição dos subtipos parasitários encontrados. Entre os indivíduos parasitados, 70,3\% estavam infectados somente por protozoários e 15,3\% infectados somente por helmintos. Houve ainda $14,2 \%$ dos indivíduos que apresentaram infecções mistas, ou seja, concomitantes por protozoários e helmintos.

Tabela 1. Prevalência global de parasitos intestinais e sua distribuição por subtipos nos indivíduos residentes na comunidade rural Rosápolis na cidade de Parnaíba, Piauí.

\begin{tabular}{lcc}
\hline \multicolumn{1}{c}{ Parasitos } & $\mathbf{N}^{\mathbf{0}}$ positivos / $\mathbf{N}^{\mathbf{0}}$ total & Freq. \% e IC95\%* \\
\hline Prevalência global & $743 / 1377$ & $53,9(51,3-56,5)$ \\
Protozoários & $523 / 743$ & $70,3(67,1-73,6)$ \\
Helmintos & $114 / 743$ & $15,3(12,7-17,9)$ \\
Infecções mistas (Ptz+Helm) & $106 / 743$ & $14,2(11,7-16,7)$ \\
\hline
\end{tabular}

* IC: Intervalo de confiança ao nível de $95 \%$.

Entre os indivíduos infectados somente por protozoários, a taxa de monoparasitismo foi de 63,2\% (IC95\%: 59,1-67,4\%), os casos de biparasitismo foram de 27,5\% (IC95\%: 23,7-31,3\%) e poliparasitismo, no qual há mais de dois diferentes parasitos, foi encontrado em 9,1\% (IC95\%: 6,7$11,6 \%$ ) dos casos (Figura 1). Entre os indivíduos infectados somente por helmintos, a taxa de 
monoparasitismo foi de 85,9\% (IC95\%: 79,5-92,3\%), enquanto os casos de biparasitismo foram de 13,1\% (IC95\%: 6,9-19,3\%) e somente um caso isolado de poliparasitismo foi encontrado representando 0,9\% (IC95\%: 0,0-2,5\%). Entre os casos de biparasitismo por helmintos, 66,6\% (IC95\%: 42,8-90,5\%) corresponderam a associações entre Ascaris lumbricoides e ancilostomídeos. Nos casos de infecções mistas, constituídas por protozoários e helmintos, a taxa de biparasitismo e poliparasitismo foi de 50,9\% (IC95\%: 41,4-60,5\%) e 49,0\% (IC95\%: 39,5-58,5\%), respectivamente. Nesta última situação relatada (infecções mistas), entre os poliparasitados foram encontrados indivíduos apresentando quatro ou cinco diferentes parasitos em 32,6\% (IC95\%: 19,9$45,4 \%)$ dos casos.

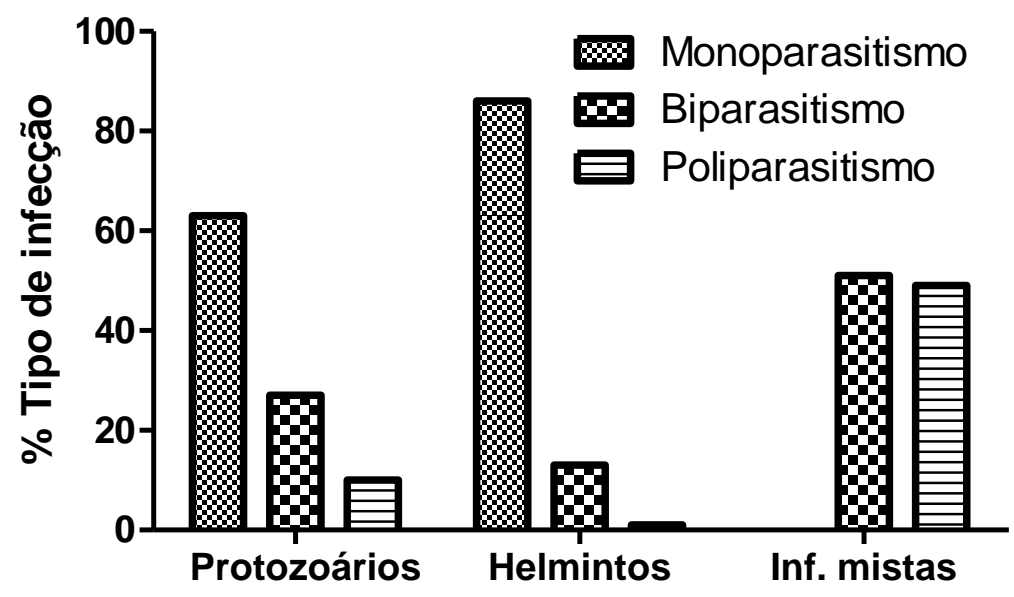

Figura 1. Distribuição e frequência das formas de parasitismo encontradas na população estudada em relação às classes de parasitos.

A infecção por protozoários apresentou maior prevalência nos indivíduos infectados (Tabela 2). Entretanto, Giardia duodenalis foi o protozoário com potencial patogênico mais frequente. Os protozoários Endolimax nana e Entamoeba coli, embora encontrados com bastante frequência, são considerados amebas comensais, sem potencial patogênico ao humano. Porém, a presença destes parasitos costuma indicar hábitos higiênico-sanitários precários [25-27].

Entre os helmintos, ovos de Ascaris lumbricoides e de ancilostomídeos foram os mais frequentes nos indivíduos infectados habitantes do referido povoado.

A prevalência global de parasitoses em relação ao sexo dos indivíduos pesquisados foi de $54,4 \%$ (IC95\%: 50,5-58,3\%) e 53,5\% (IC95\%: 49,9-57,1\%) para o sexo masculino e feminino, respectivamente. Não houve diferença estatisticamente significativa para a variável "sexo" quanto a prevalência global de parasitoses analisada pelo teste exato de Fischer ao nível de confiança de $95 \%$ ( $p$-valor $=0,744)$.

As prevalências específicas por subtipo de parasita em indivíduos do sexo masculino foram de 66,9\% (IC95\%: 64,3-69,5\%) para protozoários; 18,1\% (IC95\%: 14,0-22,2\%) para helmintos; e 14,9\% (IC95\%: 11,1-18,6\%) para infecções mistas (protozoários + helmintos). Para o sexo feminino, as prevalências específicas foram de 73,3\% (IC95\%: 68,9-77,6\%) para protozoários; 12,9\% (IC95\%: 9,6-16,2\%) para helmintos; e 13,7\% (IC95\%: 10,3-17,0\%) para infecções mistas (protozoários + helmintos). A variável "sexo" também não mostrou ter influência estatisticamente significativa sobre as prevalências específicas encontradas na população estudada quando analisada pelo teste do qui-quadrado ao nível de confiança de 95\% ( $p$-valor $=0,108$ ). 
Tabela 2. Distribuição da frequência específica dos parasitos intestinais encontrados em amostras de fezes nos indivíduos residentes na comunidade rural Rosápolis da cidade de Parnaíba, Piauí.

\begin{tabular}{lcc}
\hline \multicolumn{1}{c}{ Parasitos } & Valor absoluto $^{\#}$ & Freq. Esp. \% e IC95\%* $^{*}$ \\
\hline Endolimax nana & 381 & \\
Entamoeba coli & 267 & $51,2(48,6-53,9)$ \\
Giardia duodenalis & 190 & $35,9(32,4-39,3)$ \\
Iodamoeba butschlii & 59 & $25,6(22,4-28,7)$ \\
Entamoeba histolytica/E.dispar & 47 & $7,9(6,0-9,8)$ \\
& HELMINTOS & $6,3(4,5-8,0)$ \\
Ascaris lumbricoides & 140 & $18,8(16,0-21,6)$ \\
Ancilostomídeo & 94 & $12,6(10,2-15,0)$ \\
Trichuris trichiura & 18 & $2,4(1,3-3,5)$ \\
Hymenolepis nana & 5 & $0,7(0,1-1,3)$ \\
Strongyloides stercoralis & 4 & $0,5(0,0-1,0)$ \\
Enterobius vermicularis & 3 & $0,4(0,0-0,9)$ \\
\hline
\end{tabular}

* IC: Intervalo de confiança ao nível de 95\%. "Nesta tabela foram incluídas as infecções mistas. Assim, o valor absoluto apresenta-se maior que a prevalência global. O cálculo da porcentagem foi realizado em relação ao número total de amostras positivas $(\mathrm{n}=743)$.

A distribuição das prevalências encontradas em relação às diferentes faixas etárias dos indivíduos pesquisados foi de 46,7\% (IC95\%: 39,5-53,9\%) para indivíduos de 0 a 5 anos (valor absoluto 86/184); 60,0\% (IC95\%: 52,9-67,0\%) para indivíduos de 6 a 12 anos (valor absoluto 111/185); 55,7\% (IC95\%: 47,7-63,8\%) para indivíduos de 13 a 18 anos (valor absoluto 82/147); 52,2\% (IC95\%: 48,0-56,4\%) para indivíduos de 19 a 50 anos (valor absoluto 280/536); e 55,3\% (IC95\%: 49,4-61,2\%) para indivíduos acima de 50 anos (valor absoluto 151/273). Ressalta-se que 52 indivíduos não relataram a idade. Não houve diferença estatisticamente significativa para a variável "idade" analisada pelo teste do qui-quadrado ao nível de confiança de 95\% ( $p$-valor = $0,110)$. Verificou-se que para cada faixa etária, a prevalência global variou de $46,7 \%$ para crianças de 0-5 anos até 60,0\% para crianças de 6-12 anos. De modo geral, a prevalência global de enteroparasitoses na população do povoado se situou ao redor dos 50,0\% independentemente da faixa etária.

A partir da avaliação das respostas dadas ao questionário socioeconômico e higiênico-sanitário, que estão detalhadas na Tabela 3, foi verificado um percentual de indivíduos não alfabetizados de $19,2 \%$, enquanto $61,4 \%$ e $18,8 \%$ relataram ter alcançado o ensino fundamental e médio, respectivamente. Entre as residências pesquisadas, $94,2 \%$ são abastecidas por fornecimento público de água. Essa mesma água que chega às residências é utilizada para o consumo humano em 47,2\% das residências, enquanto que em $51,4 \%$ das residências, o consumo da água é feito com o acréscimo de algum tratamento como filtração ou fervura.

Em relação às formas de higienização dos alimentos, em 57,5\% das residências houve o relato de uso da água da torneira para lavagem de frutas e verduras, enquanto que em 16,1\% das residências foi relatado a não higienização destes alimentos. Neste mesmo aspecto, o consumo de verduras cruas foi um hábito comum relatado em $69,7 \%$ das residências.

Em relação ao destino dado ao lixo domiciliar e/ou resíduos caseiros descartados, em 48,3\% das residências foi relatada a "queima" do lixo, enquanto que em 33,3\% das residências houve o relato do lixo ser periodicamente recolhido. 
Tabela 3. Aspectos socioeconômicos e higiênico-sanitários relatados pelos indivíduos responsáveis pelas residências que participaram do estudo $(n=360)$.

\begin{tabular}{lcc}
\hline Característica & $\mathbf{N}^{\mathbf{0}}$ de relatos & Freq. \% e IC95\%* \\
\hline Nível de escolaridade & 69 & $19,2(16,5-21,8)$ \\
Não alfabetizado & 221 & $61,4(56,4-66,4)$ \\
Ensino fundamental (completo/incompleto) & 68 & $18,8(14,8-22,9)$ \\
Ensino médio (completo/incompleto) & 02 & $0,6(0,0-1,3)$ \\
Ensino superior & & \\
Abastecimento de água & 339 & $94,2(91,7-96,6)$ \\
Rede pública & 18 & $5,0(2,7-7,3)$ \\
Poço ou cisterna & 03 & $0,8(0,0-1,8)$ \\
Água de rio & & \\
Água de consumo & 185 & $51,4(46,2-56,6)$ \\
Filtrada/Fervida & 170 & $47,2(42,1-52,4)$ \\
Água da torneira/sem tratamento & 05 & $1,4(0,2-2,6)$ \\
Mineral & & \\
Destinação do lixo & 120 & $33,3(28,5-38,2)$ \\
Recolhido & 174 & $48,3(43,2-53,5)$ \\
Queimado & 41 & $11,4(8,1-14,7)$ \\
Enterrado & 25 & $7,0(4,3-9,6)$ \\
Depositado no meio ambiente & & \\
Consumo de verduras cruas & 250 & $69,7(64,7-74,2)$ \\
Sim & 110 & $30,6(25,8-35,3)$ \\
Não & & \\
Água usada na higienização de frutas e/ou verduras & 207 & $57,5(52,4-62,6)$ \\
Água da torneira & 84 & $23,3(19,0-27,7)$ \\
Água com tratamento químico (NaClO) & 11 & $3,1(1,3-4,8)$ \\
Água filtrada/fervida & 58 & $16,1(12,3-19,9)$ \\
Sem higienização & &
\end{tabular}

* Intervalo de confiança ao nível de $95 \%$.

\section{DISCUSSÃO}

Ainda que as condições de saneamento básico e hábitos higiênico-sanitários tenham passado por substanciais e visíveis melhorias nos últimos anos, particularmente nas cidades mais desenvolvidas, fato este que tem reduzido a prevalência das parasitoses intestinais [27-29], essas infecções ainda atingem muitos indivíduos, especialmente crianças [30], adolescentes [23], idosos [17] e moradores de regiões economicamente carentes localizadas nas periferias dos grandes centros [9, 22, 31-32]. É importante ressaltar que as infecções por enteroparasitos possuem tratamento rápido, eficiente e de baixo custo [33]. Entretanto, quando não há mudanças nos hábitos que viabilizam as transmissões, as reinfecções ocorrem frequentemente garantindo suas elevadas prevalências [23].

Indivíduos residentes em povoamentos isolados, tais como aldeias, comunidades rurais, povoados e assentamentos, estão entre os mais vulneráveis às contaminações por infecções parasitárias e também à sua manutenção, pois nestes locais, o diagnóstico nem sempre é feito regularmente, retardando o tratamento adequado, e causando a persistência da cadeia de infecção. Em termos de prevalência global, os resultados desse presente estudo corroboram com os encontrados por Alves et al. (2003) [32] que analisaram amostras de fezes de indivíduos residentes no Município de São Raimundo Nonato, localizado a $520 \mathrm{~km}$ de Teresina, região semi-árida do estado do Piauí, encontrando prevalência global de $57 \%$ de parasitados. Entretanto, por se tratar de populações que vivem em condições precárias de saneamento básico, prevalências ainda maiores 
poderão ser encontradas, como as relatadas por Arias e Urrego (2013) [9] que realizaram estudo epidemiológico em uma comunidade periférica da cidade de Medellín, Colômbia, e encontraram uma prevalência global de 74,4\%; Silva et al. (2012) [11] que analisaram amostras fecais de crianças de uma comunidade ribeirinha no município de Coari, Amazonas, encontrando prevalência de 83,1\%; Ferreira et al. (2002) [2] que analisaram amostras de fezes de crianças residentes em um assentamento de movimento social com condições de "extrema pobreza" e encontraram uma prevalência global de 83,2\%; e Escobar-Pardo et al. (2010) [8] que analisaram amostras de fezes de crianças de seis aldeias do baixo Xingu encontrando prevalência de 96,1\%. Esses valores corroboram a existência de alarmantes prevalências de infecções parasitárias nestas regiões.

Por outro lado, municípios com melhores taxas de desenvolvimento social e urbano têm apresentado valores menores de prevalência de parasitoses intestinais, por exemplo, os municípios de Bom Jesus dos Perdões e Assis, ambos no estado de São Paulo, que segundo estudos realizados por Lodo et al. (2010) [27] e Frei et al. (2008) [29], apresentaram prevalências de parasitos intestinais de $15,7 \%$ e $20,3 \%$, respectivamente. Para efeitos de comparação de desenvolvimento urbano, o IDHM (Índice de Desenvolvimento Humano Municipal) do município de Assis é de 0,805 e do município de Bom Jesus dos Perdões é de 0,713, enquanto o município de Parnaíba, onde o presente estudo foi conduzido, apresenta IDHM de 0,687 [34]. Porém, quando se trata de populações mais suscetíveis, como as crianças, mesmo em cidades com maiores IDHM as prevalências também podem ser altas. O estudo conduzido por Biasi et al. (2010) [7] com crianças de uma entidade assistencial no município de Erechim (RS), que apresenta IDHM de 0,776, mostrou prevalência de parasitoses de 54,5\%. O estudo de Reuter et al. (2015) [30] com crianças de creches no município de Santa Cruz do Sul (RS) revelou uma prevalência de 32,3\%, mesmo apresentando um IDHM de 0,773. Essas situações de valores discrepantes de prevalência revelam como a avaliação dos fatores socioeconômicos em relação ao risco de infecções por parasitos intestinais é uma variável complexa de ser estudada. Além disso, pode-se inferir a importante e significativa parcela de contribuição que a educação sanitária da população tem na redução das infecções por estes agentes preveníveis [20], embora alguns estudos têm também revelado que a educação sanitária sozinha não seja eficiente para manter taxas de prevalência reduzidas [26].

Dentre os parasitos encontrados, verifica-se que Giardia duodenalis e Ascaris lumbricoides, respectivamente nas classes de protozoários e helmintos, correspondem às principais espécies patogênicas encontradas em estudos epidemiológicos desta natureza nas cidades brasileiras [7, 3031]. Mais uma vez, é importante ressaltar que as espécies comensais, não patogênicas, como Entamoeba coli e Endolimax nana também foram relatadas em diversos outros estudos e estão relacionadas aos indicadores de contaminação oral-fecal, visto que em geral, esses agentes compartilham formas similares de transmissão [25-28].

Foram verificados poucos casos de Enterobius vermicularis e outros helmintos de formas larvais e da classe cestoide. Silva e Silva (2010) [35] encontraram resultados similares e os atribuem a uma possível subestimação causada pelo uso de uma metodologia não específica para esses parasitos. No presente estudo, também não foi utilizada metodologia específica para detecção de formas larvais, embora elas também possam ser detectadas pela metodologia da sedimentação espontânea aplicada.

Em relação às variáveis sexo e faixa etária, verificou-se que não houve diferença estatisticamente significativa entre os grupos na referida população. Alguns estudos epidemiológicos sobre parasitoses intestinais têm mostrado que o sexo feminino geralmente apresenta maior prevalência, embora as diferenças encontradas não tenham sido estatisticamente significativas em alguns casos $[10,12,27]$. Situação similar também foi encontrada em relação à variável "faixa etária" dos indivíduos pesquisados. Pode-se dizer, de maneira geral, que foi observado que metade dos indivíduos em todas as diferentes faixas etárias estava infectada por algum parasito. Alguns outros estudos relatam prevalências diferentes em determinadas faixas etárias, por exemplo, Ferreira et al. (2002) [2] e Souza et al. (2016) [13] analisaram amostras de fezes de um assentamento de movimento social e um assentamento rural no nordeste brasileiro, respectivamente, e encontraram prevalências significativamente maiores em indivíduos com idade acima de 45 anos.

$\mathrm{Na}$ avaliação dos aspectos socioeconômicos da população estudada, o baixo nível de escolaridade relatado pode explicar a alta prevalência das parasitoses encontradas [5]. Outros 
autores têm relacionado a baixa escolaridade com menor conhecimento específico sobre os riscos de transmissão e as formas de eliminação dos parasitos [20]. Entretanto, o saber "senso comum" a respeito da cadeia de transmissão dessas doenças, sem que isso gere uma mudança prática dos hábitos, não impede a continuidade das infecções na população. Além disso, como consequência da pouca escolaridade da comunidade, surgem as baixas condições econômicas que atuam como fatores complementares de risco para a ocorrência e a persistência das enteroparasitoses. O estudo de Busato et al. (2015) [20] relata de forma mais aplicada a correlação do conhecimento popular com os riscos de transmissão dos parasitos.

Quanto aos hábitos de consumo, tais como os cuidados adequados para o tratamento da água e higienização dos alimentos, sabe-se que esses fatores atuam como importantes condições que possibilitam a transmissão das principais espécies de geohelmintos e protozoários de veiculação hídrica $[3,19]$. Outras variáveis, tais como a contaminação ambiental, o depósito inadequado do lixo doméstico, a precariedade do saneamento básico e outros hábitos higiênico-sanitários inadequados também estão presentes em relatos coletados em questionários socioepidemiológicos aplicados aos indivíduos residentes em diferentes regiões estudadas [6, 13, 19, 22, 36]. Tais condições formam uma situação ecoepidemiológica que culmina no favorecimento da transmissão e reinfecção de doenças infecto-parasitárias, em especial helmintos e protozoários, explicitando a semelhança na alta prevalência dessas doenças tanto em zonas rurais como urbanas $[19,13,27$, 37].

Ainda que não tenha sido possível comprovar nesse estudo associações estatisticamente significativas entre prevalência das parasitoses e as variáveis sociais e higiênico-sanitárias, sabe-se que esses são fatores historicamente importantes que aumentam o risco de transmissão das infecções e propiciam sua manutenção nas comunidades estudadas. Há de se ressaltar que pessoas parasitadas sem tratamento são veículos de disseminação, porém a transmissão oral-fecal de enteroparasitos ocorre principalmente por meio de um ambiente favorável à manutenção dos parasitos em estado viável, tais como nos solos e ambientes aquáticos. O ambiente rural frequentemente apresenta condições adversas, tais como ausência de pavimentação, saneamento básico precário, sistema de esgoto inexistente, água empoçada em ruas, animais abandonados sem tratamento antiparasitário, coleta de lixo irregular e poluição ambiental por depósito de lixo. Essas condições, aliadas ao inadequado tratamento da água para consumo e para higienização efetiva de frutas e verduras, e a falta de educação higiênico-sanitária, propiciam a manutenção interna do ciclo de infecção. Ou seja, os indivíduos continuam se contaminando por parasitos no próprio ambiente onde vivem, uma vez que nesses povoados, o deslocamento para áreas urbanas é realizado apenas ocasionalmente.

As informações de prevalência e fatores de risco têm bastante relevância à saúde das populações alvo desses estudos, pois ressalta a urgente necessidade de tomada de decisões governamentais e sociais no sentido de eliminar as fontes de infecção e impedir a propagação das doenças infectoparasitárias. Diversas iniciativas podem fazer surtir efeitos benéficos para a redução das prevalências de infecções parasitárias, tais como intervenções educativas permanentes [26] e tratamentos profiláticos controlados [29, 33].

\section{CONCLUSÃO}

Com base nos resultados apresentados, verificou-se uma alta prevalência de parasitoses intestinais humanas na comunidade rural de Rosápolis, localizada no município de Parnaíba, estado do Piauí. Neste povoado, a prevalência de enteroparasitoses foi similar entre homens e mulheres, independentemente da faixa etária. Houve relatos de hábitos higiênico-sanitários que são tradicionalmente relacionados à manutenção do ciclo de infecção parasitária. Portanto, as iniciativas governamentais no sentido de implantar melhorias nas condições básicas de educação e saneamento, além da conscientização populacional dos riscos de transmissão das enteroparasitoses poderão diminuir as taxas de infecção, garantindo uma melhor condição de vida em termos de ausência de agentes patogênicos preveníveis, para a referida população. 


\section{AGRADECIMENTOS}

Os autores agradecem ao $\mathrm{CNPq} /$ Brasil pelo suporte financeiro obtido por meio do projeto Universal (processo nº 476396/2011-5), à Universidade Federal do Piauí, campus de Parnaíba, pelo apoio estrutural para a realização das análises.

\section{CONFLITO DE INTERESSE}

Os autores declaram não haver conflito de interesse no desenvolvimento deste estudo.

\section{REFERÊNCIAS BIBLIOGRÁFICAS}

1. Drake LJ, Bundy DAP. Multiple helminth infections in children: impact and control. Parasitology. 2001,122(S1):S73-S81, doi:10.1017/S0031182000017662.

2. Ferreira HS, Assunção ML, Vasconcelos VS, Melo FP, Oliveira CG, Santos TO. Saúde de populações marginalizadas: desnutrição, anemia e enteroparasitoses em crianças de uma favela do "Movimento dos Sem Teto", Maceió, Alagoas. Rev Bras Saúde Mater Infant. 2002,2(2):177-185, doi:10.1590/S151938292002000200010

3. Brooker S, Clements ACA, Bundy DAP. Global Epidemiology, Ecology and Control of Soil-Transmitted Helminth Infections. Adv Parasitol. 2006,62:221-261, doi: 10.1016/S0065-308X(05)62007-6.

4. Ezeamama AE, Friedman JF, Acosta LP, Bellinger DC, Langdon GC, Manalo DL, Olveda RM, Kurtis JD, Mcgarvey ST. Helminth infection and cognitive impairment among Filipino children. Am J Trop Med Hyg. 2005,72(5):540-548.

5. Ferreira GR, Andrade CFS. Alguns aspectos socioeconômicos relacionados a parasitoses intestinais e avaliação de uma intervenção educativa em escolares de Estiva Gerbi, SP. Rev Soc Bras Med Trop. 2005,38(5):402-405, doi:10.1590/S0037-86822005000500008.

6. Nolla AC, Cantos GA. Relação entre a ocorrência de enteroparasitoses em manipuladores de alimentos e aspectos epidemiológicos em Florianópolois, Santa Catarina, Brasil. Cad Saúde Pública. 2005,21:34-45, doi: $10.1590 / \mathrm{S} 0102-311 \mathrm{X} 2005000200033$.

7. Biasi LA, Tacca JA, Navarini M, Belusso R, Nardino A, Santolin JC, Bernardon V, Jaskulski MR. Prevalência de enteroparasitoses em crianças de entidade assistencial de Erechim/RS. Perspectiva. 2010,34(125):173-179.

8. Escobar-Pardo ML, de Godoy AP, Machado RS, Rodrigues D, FagundesNeto U, Kawakami E. Prevalence of intestinal parasitoses in children at the Xingu Indian Reservation. J Pediatr (Rio J). 2010,86(6):493-496, doi:10.2223/JPED.2036.

9. Arias JAC, Urrego KB. Frecuencia de parásitos intestinales y evaluación de métodos para su diagnóstico en una comunidad marginal de Medellín, Colombia. Iatreia. 2013,26(3):257-268.

10. Andrade EC, Leite ICG, Vieira MT, Abramo C, Tibiriçá SHC, Silva PL. Prevalência de parasitoses intestinais em comunidade quilombola no Município de Bias Fortes, Estado de Minas Gerais, Brasil, 2008. Epidemiol Serv Saúde. 2011,20(3):337-344, doi:10.5123/S1679-49742011000300008.

11. Silva EF, Silva VBC, Freitas FLC. Parasitoses intestinais em crianças residentes na comunidade ribeirinha São Francisco do Laranjal, município de Coari, Estado do Amazonas, Brasil. Rev Patol Trop. 2012,41(1):97-101, doi:10.5216/rpt.v41i1.17753.

12. Assis EM, Olivieria RC, Moreira LE, Pena JL, Rodrigues LC, Machado-Coelho GLL. Prevalência de parasitos intestinais na comunidade indígena Maxakali, Minas Gerais, Brasil, 2009. Cad Saúde Pública. 2013,29(4):681-690, doi: 10.1590/S0102-311X2013000400006.

13. Souza AC, Alves FV, Guimarães HR, Amorim ACS, Cruz MA, Santos BS, Borges EP, Trindade RA, Melo ACFL. Perfil epidemiológico das parasitoses intestinais e avaliação dos fatores de risco em indivíduos residentes em um assentamento rural do nordeste brasileiro. Rev Conexão UEPG. 2016,12(1):26-37, doi: 10.5212/Rev.Conexão.v.12.i1.0002

14. Instituto Brasileiro de Geografia e Estatistica. Indicadores Sociodemográficos e de Saúde no Brasil. In: Estudos e Pesquisas Informação Demográfica e Socioeconômica, número 25. Rio de Janeiro: IBGE; 2009.

15. Muniz-Junqueira MI, Queiróz EFO. Relationship between protein-energy malnutrition, vitamin A, and parasitoses in children living in Brasília. Rev Soc Bras Med Trop. 2002,35(2):133-141, doi:10.1590/S0037-86822002000200002.

16. Hechenbleikner EM, McQuade JA. Parasitic Colitis. Clin Colon Rectal Surg. 2015,28:79-86, doi:10.1055/s-0035-1547335. 
17. Furtado LFV, Melo ACFL. Prevalência e aspectos epidemiológicos de enteroparasitoses na população geronte de Parnaíba, estado do Piauí. Rev Soc Bras Med Trop. 2011,44(4):513-515, doi:10.1590/S003786822011000400023.

18. Melo ACFL, Ceia-Junior EA, Azevedo IM, Souza PDA, Miranda CRL, Borges EP, Trindade RA. Aspectos epidemiológicos das enteroparasitoses em crianças de uma unidade pública de ensino de Parnaíba, Piauí. Unopar Cient Ciênc Biol Saúde. 2014,16:191-196.

19. Fernandes NS, Guimaraes HR, Amorim ACS, Brito VM, Borges EP, Reis, MB, Trindade RA, Melo ACFL. Ocorrência de enteroparasitoses em manipuladores de alimentos de restaurantes em Parnaíba, Piauí-Brasil. Rev Patol Trop. 2015,43:459-469, doi:10.5216/rpt.v43i4.33614.

20. Busato MA, Dondoni DZ, Rinaldi ALS, Ferraz L. Parasitoses intestinais: o que a comunidade sabe sobre este tema? Rev Bras Med Fam Comunidade. 2015,10(34):1-6, doi:10.5712/rbmfc10(34)922.

21. Zaiden MF, Santos BMO, Cano MAT, Júnior IAN. Epidemiologia das parasitoses intestinais em crianças de creches de Rio Verde-GO. Medicina (Ribeirão Preto). 2008,41(2):182-7.

22. Oliveira CLM, Ferreira WA, Vasquez FG, Barbosa MGV. Parasitoses intestinais e fatores socioambientais de uma população da área periurbana de Manaus - AM. Rev Bras Promoç Saúde. 2010,23(4):307-315, doi:10.5020/18061230.2010.p30.

23. Belo VS, Oliveira RB, Fernandes PC, Nascimento BWL, Fernandes FV, Castro CLF, Santos WB, Silva ES. Factors associated with intestinal parasitosis in a population of children and adolescents. Rev Paul Pediatr. 2012,30(2):195-201, doi:10.1590/S0103-05822012000200007.

24. Hoffman WA, Pons JA, Janer JL. Sedimentation concentration method in schistosome. The Puerto Rico Journal of Public Health and Tropical Medicine. 1934,9(3):283-91.

25. Macedo HS. Prevalência de Parasitos e comensais intestinais em crianças de escolas da rede pública municipal de Paracatu (MG). Rev Bras Anal Clin. 2005,37(4):209-13.

26. Carvalho-Haesbaert T, Lamas RH, Novaes-Júnior DF, Hauck da Silva A, Evangelista MGBF. Avaliação do impacto de uma intervenção educativa na ocorrência de enteroparasitoses em escolares no município de Jundiaí, SP. Perspectivas Médicas. 2009,20(2):10-15.

27. Lodo M, Oliveira CGB, Fonseca ALF, Caputto LZ, Packer MLT, Valenti VE, Fonseca FLA. Prevalência de enteroparasitas em município do interior paulista. Rev Bras Crescimento Desenvolvimento Hum. 2010, 20(3):769-777.

28. Basso RMC, Silva-Ribeiro RT, Soligo DS, Ribacki SI, Callegari-Jacques SA, Zoppas BCA. Evolução da prevalência de parasitoses intestinais em escolares em Caxias do Sul, RS. Rev Soc Bras Med Trop. 2008,41(3):263-268, doi:10.1590/S0037-86822008000300008.

29. Frei F, Juncansen C, Ribeiro-Paes JT. Levantamento epidemiológico das parasitoses intestinais: viés analítico decorrente do tratamento profilático. Cad Saúde Pública. 2008,24(12):2919-2925, doi:10.1590/S0102-311X2008001200021.

30. Reuter CP, Furtado LBFS, Silva R, Pasa L, Klinger EI, Santos CE, Renner JDP. Frequência de parasitoses intestinais: um estudo com crianças de uma creche de Santa Cruz do Sul - RS. Cinergis. 2015,16(2):142147, doi:10.17058/cinergis.v16i2.6426.

31. Uchôa CMA, Lobo AGB, Bastos OMP, Matos AD. Parasitoses intestinais: prevalência em creches comunitárias da cidade de Niterói, Rio de Janeiro - Brasil. Rev Inst Adolfo Lutz. 2001,60(2):97-101.

32. Alves JR, Macedo HW, Ramos Jr AN, Ferreira LF, Gonçalves MLC, Araújo A. Parasitoses intestinais em região semi-árida do Nordeste do Brasil: resultados preliminares distintos das prevalências esperadas. Cad Saúde Pública. 2003,19(2):667-670, doi:10.1590/S0102-311X2003000200034.

33. Lo NC, Bogoch II, Blackburn BG, Raso G, N'Goran EK, Coulibaly JT, Becker SL, Abrams HB, Utzinger J, Andrews JR. Comparison of community-wide, integrated mass drug administration strategies for schistosomiasis and soil-transmitted helminthiasis: a cost-effectiveness modelling study. The Lancet. Global health. 2015,3(10):e629-38, doi:10.1016/S2214-109X(15)00047-9.

34. PNUD. Programa das Nações Unidas para o Desenvolvimento. Atlas do Desenvolvimento Humano, 2013. Acesso em 09Fev2017. Disponível em <http://www.atlasbrasil.org.br/2013/>.

35. Silva LP, Silva RMG. Ocorrência de enteroparasitos em centros de educação infantil no município de Patos de Minas, MG, Brasil. Biosci J. 2010,26(1):147-151.

36. Komagome SH, Romagnoli MPM, Previdelli ITS, Falavigna DLM, Dias MLGG, Gomes ML. Fatores de risco para infecção parasitária intestinal em crianças e funcionários de creche. Cienc Cuid Saude. 2007,6(S2):442-447, doi:10.4025/cienccuidsaude.v6i0.5354.

37. Santos SA, Merlini LS. Prevalência de enteroparasitoses na população do município de Maria Helena, Paraná. Ciênc Saúde Coletiva. 2010,15(3):899-905, doi:10.1590/S1413-81232010000300033. 